\title{
Computer Numerical Control System for Automatic Surface Machining
}

\author{
Chen Zuo Yue, Wang Xiao E, Yang Mei \\ Department of Electromechanical Engineering, Leshan Vocational and Technical College, Leshan \\ 614000, China \\ chengzuoy@tom.com
}

\begin{abstract}
Keywords: Automatic Control, CNC Machining, Control Engineering, Engineering Design
\end{abstract}
\begin{abstract}
Computer numerical control technology is mechanical movement with digital information and work process control technology, data processing is the reverse engineering of important technical aspects, which determines whether the subsequent model reconstruction process easy and accurate manner. In this paper, computer numerical control machining complex surfaces feature full potential of existing computer numerical control equipment, automatic control system designed to make computer numerical control machining equipment to achieve higher processing speed, high precision machining, and computer numerical control equipment in order to achieve high efficiency machining.
\end{abstract}

\section{Introduction}

Computer numerical control machining process design is a core part of the NC programming, only the computer numerical control machining process is reasonable, scientifically into NC programming, will it be possible to get a high quality and high levels of the NC program. The computer numerical control process is improvement programs. The purpose of computer numerical control machining process designed to determine the processing method, designated processing program, as well as issues related to recognition and production organization. The main contents include selection processing program; determine the processing order, processing routes; determine positioning and clamping program parts; select the appropriate tool and cutting parameters; arrange heat treatment, inspection and auxiliary processes, etc.; while also considering the use of computer numerical control machine tools function, and give full play to the performance of the machine. Processing routes as short as possible, under normal circumstances should be more developed several programs, analysis and comparison, choose the most economical solution.

Because of geometric constraints exist between various types of surface data points, thus making optical designers get great design freedom in the design, which means that if the optical system into the free-form optical components, optical performance can achieve more excellent (such as correcting aberrations, expanding the field of view, increasing the role of distance, improve image quality), and the plurality of spherical, spherical lens may consist of one or a few free-form surface mirror instead, so as to achieve weight reduction, reduce costs and simplify the optical system configuration purposes. Free-form surface is an irregular, complex, non-rotary surface, it is difficult to accurately describe mathematical expressions, generally use a large number of discrete data points to describe. For every point, there is always a maximum normal curvature and a minimum normal curvature surface. Set the maximum principal curvatures $k_{1}, k_{2}$ is the minimum principal curvature, principal curvatures calculated according to the Gaussian curvature $K$ and mean curvature $H$ are:

$$
\left\{\begin{array}{l}
K=k_{1} \cdot k_{2} \\
H=\frac{k_{1}+k_{2}}{2}
\end{array}\right.
$$

According symbol Gaussian curvature $\mathrm{K}$ and mean curvature $\mathrm{H}$ eight curved shape can be determined: peak surface, ridge surface, saddle ridge, flat, minimal surfaces, concave surface and saddle valley. Gaussian curvature is defined as the point on the surface of the maximum principal 
curvatures $\mathrm{k} 1$ and $\mathrm{k} 2$ minimum principal curvature of the plot, the surface can reflect the degree of deformation and distortion degree in two main directions, often in hazardous areas Gaussian curvature surface machining of larger value. Machining complex surfaces are positioned first installation, installation is necessary to make allowance positioned evenly as possible; the second is to minimize the processing workload of complex surfaces, in order to improve the processing efficiency of the complex surface.

\section{Positioning Complex Surface Machining}

Surface matching is actually two representations of the same surface in two different coordinate systems in space alignment process. When the three-axis machining rough underside is coarse positioning reference to processing underside error and positioning error due to the coarse positioning reference by foam machining errors caused by the installation of casting and casting time constrained, while simultaneously casting mold surface deformation, this has led to the car cover when machining mold surface, allowance is uneven, thereby forming a plurality of tool air travel, wasting a lot of man-hours.

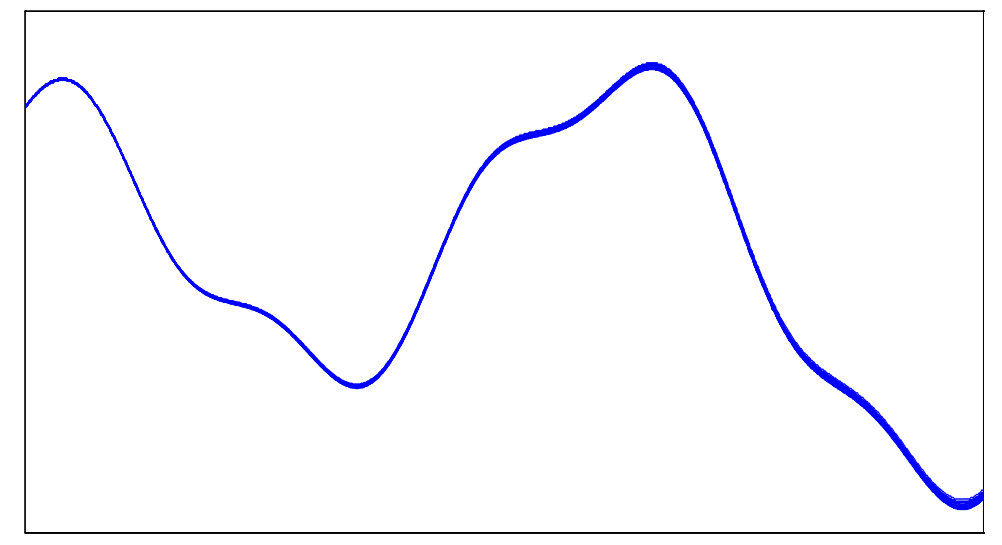

Figure.1 allowance due to positioning causes uneven schematic

In medium-sized surface mold CNC machining CNC machining processing time occupied the majority, therefore, should be to ensure uniform mold surface and processing allowance for the guidelines to improve the processing efficiency of CNC machine tools, positioning must be installed taking into account the limiting block mounting surface or safety surface and a bottom surface, such as machined surface machining datum, to ensure that they have sufficient surface to be machined allowance. When mold casting, casting figure is based on an offset section reserved for allowance on the basis of the product obtained in Fig. For a flat surface to be machined, complex curved surface to be machined, cast figure is offset along the surface normal for some complex allowance figure obtained by the product or the use of z-directional shift.

Machine bed, column, table, beams, box, base and other supporting large, its body structure has the following two characteristics: the physical point of view, the structure of space plate line; from the load point of view, the spatial force Department carrier. Machine support large panels belong to space architecture or plates, beams and other space composite structure. The machine can produce various forces in static and dynamic medium, directly or indirectly, by the large to bear. They are cutting force, gravity, friction, clamping force, inertial force and shock or vibration disturbance force. Machine bulky design, the various forces usually summarized as static force and dynamic force, and then were studied static stiffness and dynamic stiffness. Under the large, heavy, bulky high-speed precision machine tools, but also taking into shock or vibration disturbance force, may produce displacement, velocity and acceleration, as well as due to the emergence of structural instability siding sectional shape distortion and noise, dynamic response issues. For high-precision machine tools, such as grinders, because the process produces a large amount of cutting heat, heat the spindle 
motor, and therefore also take into account the thermal deformation of large pieces. Therefore, the support of the design requires large force, deformation and stiffness analysis.

\section{CNC Design Process Scheme}

Based on the above ideas, the positioning of the work piece into the work piece blank point measurement data and CAD models to the difference between the square on the plane and take the minimum distance problem. To make the point corresponding point on the CAD model and rough on the surface matched to establish rigid motion transformation between surface measurement coordinate system and design coordinate system. Evaluation process, to ensure that each point corresponding to the scanning point on the work piece blank coordinate transformation, making the scanning point on the work piece blank limiting block mounting surface and CAD models only parallel to the upper surface of the block is installed with the machine the face, and then seeking point blank type mold surface to limiting the distance the plane on the block mounting surface.

Processing coordinate system is the processing origin as a reference coordinate system established. The role of processing coordinate system that is defined geometry processing on CNC machine location coordinates of the tool path programming is to press the part contour machining coordinate system in the coordinate determined. In the process, the CNC machine is determined in accordance with the processing of the workpiece clamping position of the origin and procedural requirements for processing.

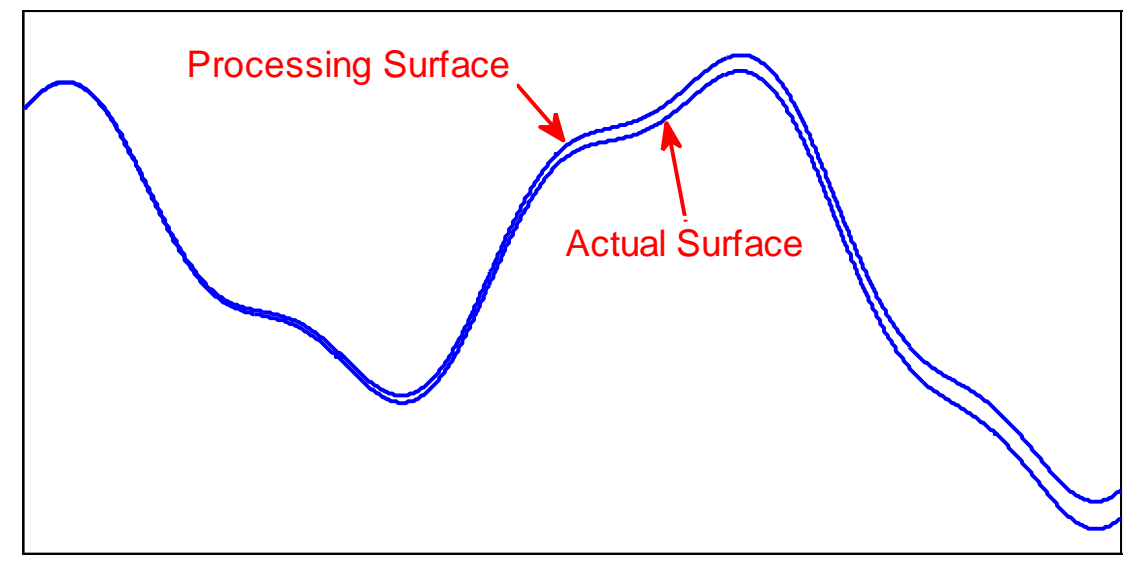

Figure.2 blank design surface \& real surface to be processed

UG provides a powerful set of coordinates function, ACS (absolute coordinate system), WCS (work coordinate system), and MCS (machining coordinate system) and RCS reference coordinate system. 3 axis machining coordinate system respectively XM, YM, ZM, the default tool axis vector direction for ZM, you can additionally specify the tool axis vector direction, the origin of the coordinate system that is processing the knife point on the machine, three direction is the direction of the axis machine tool guide, so when deciding on the location of the origin and direction of machining coordinate system, the actual processing conditions should proceed to ensure that rough location on the machine's ease of setup, processing and knife.

Total flexibility in machine flexibility than the system components to the largest, which means the overall stiffness of the machine rigidity than the system in the worst parts is smaller. Therefore, to improve the overall stiffness of the design of the machine, the system must pay particular attention to structural design weaknesses, including large contact stiffness between. In order to meet the high productivity CNC machining, high speed, high precision, high reliability and high degree of automation requirements, CNC machine tools generally considered to be higher than the stiffness of at least 50 percent more than conventional machine tools. Here the use of approximation method is approximately equal to the iterative approach kept the coefficients, because the system is broken down into many songs unit, relying on a number of key factors linked between units, thus obtaining a pluralistic multi unknowns equation Solutions group, are a function of each equation, as many 
equations, the decomposition of the problem is very close to the solution of the system. As a result of the problem solving system transformed into a multi-function equation issue, saying the project is seeking a number of multi-freedom issues field function.

In the processing, the selected motion relative to the product line is called the tool pass mode, the machining accuracy of its choice will be the product of cutting efficiency, interference produced, production costs low, even CNC machine tool life and sports performance are has important implications. This method is commonly used in the processing of complex irregular surface, the tool paths are flat trajectory parallel to each other, are straight, clear and intuitive, and the resulting small CNC program, each program segment length is long, the cutting efficiency greatly improved. The model is divided into one-way pass mode, take the knife back and forth pattern two classes (Fig.3).

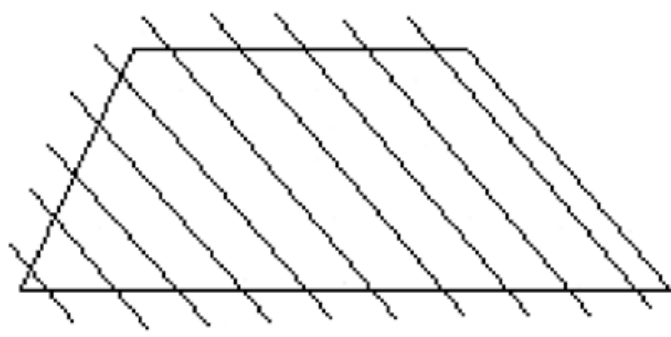

Figure.3 (a) Straight away

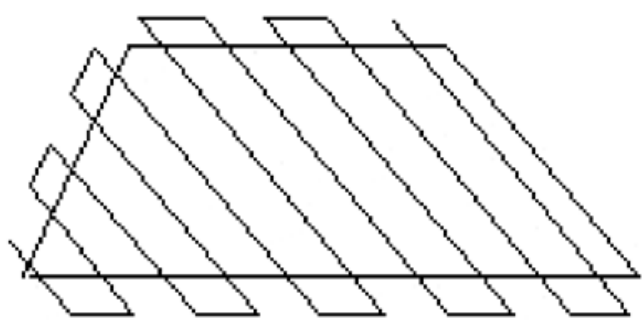

(b) Round-trip away

One-way pass mode enables optimal tool machining direction along the road, after processing the resulting surface quality is excellent, but for repeatedly mentioning the knife, there is a lot of air travel, the efficiency is not high natural; take the knife from the direction of travel modes and cutting forces are constantly repeated changes in direction, the impact on the larger machines, poor quality of the resulting surface, but the efficiency is relatively high.

The content can not be used as a control command of CNC machine tools, CNC system due to various CNC machine tools being used are not the same, for the same function in different CNC system whose code is not exactly the same, so the file must be converted to a cutter path CNC machine tools to meet specific requirements of the Directive and processing procedures. In NC programming, through post-processing operations knife-bit track file into the machine controller can be recognized by the NC code. Now commonly used in CAD / CAM software is usually set up a post-processing using the file, before performing post-processing, the programmer simply good this file format specific CNC machine according to the instruction code and procedures prior to edit, and then on the cutter path file post-processing can be obtained NC machining CNC machining format files that meet the requirements. Achieved using UG / CAM module compiled mouse parts CNC machining program, presented the overall shape of the part cavity milling roughing, finishing on the mouse surface fixed axis curved contour, contour milling contour machining strategies side of the mouse, and in accordance with the policy NC programming work done, and finally the use of post-processing module generates a specific machine control system that can be recognized by the NC code as the basis for CNC machining simulation work.

\section{Conclusion}

Surface quality testing is an important means to judge the merits of the surface. Paper to measure the distance to the surface model as the main evaluation index, respectively, for the two surface model shoe traditional surface modeling methods and fast way to get surface modeling for surface evaluation, advantages and disadvantages, and the conclusions drawn are two ways of surface reconstruction : when asked curved stitching no obvious signs of overall relatively smooth surfaces or require shorter reconstructed surface, the surface structure can abandon traditional methods, choose fast surface modeling methods; when asked at the curvature of the surface changes in the larger error than the when a small or local surface high precision, fast surface modeling can give way, choose the traditional surface modeling methods. 
Complex surfaces in industrial applications more widely. In this paper, the research group previously designed two types of large, complex surfaces CNC milling machine. Due to the large size of the workpiece, the design of the machine structure size of up to 30 meters, poor machine stiffness. This paper designed a mobile column milling machine arm. The overall layout of the form to start from structural design, design a high rigidity pendulum rotary power head and internal organs do static stress and displacement analysis.

\section{Acknowledgements}

The authors thank the Natural Science Foundation of Sichuan Education Department (project number: 14ZB0394).

\section{References}

[1] K. Fan, J. Yang, L. Yang: Mechanical Systems and Signal Processing, Vol.49 (2014), p.63-76

[2] F. J. Meng, Z. T. Chen, R. F. Xu, X. Li: Computer-Aided Design, Vol.53 (2014), p.36-45

[3] S. L. Omirou: Robotics and Computer-Integrated Manufacturing, Vol.20 (2004), p.255-264

[4] C. H. Liu, A. Chen, C. C. A. Chen, Y. T. Wang: Journal of Materials Processing Technology, Vol.170(2005), p.367-373

[5] F. Ridwan, X. Xu: Robotics and Computer-Integrated Manufacturing, Vol.29 (2013), p.12-20

[6] M. I. Gökler, M. B. Koç: International Journal of Machine Tools and Manufacture, Vol.37 (1997), p.277-286

[7] X. Zhao, Y. Pan, C. Zhou, Y. Chen, C. C. L. Wang: Journal of Manufacturing Processes, Vol.15 (2013), p.432-443

[8] S. L. Omirou: Robotics and Computer-Integrated Manufacturing, Vol.20 (2004), p.49-55

[9] B. R. Borkar, Y.M. Puri, A.M. Kuthe, P.S. Deshpande: Procedia Materials Science, Vol.5 (2014), p.2513-2521 